\title{
Evidence-based medicine during the COVID-19 pandemic: a survey among healthcare professionals in India
}

\author{
Author: Vinayak Mishra ${ }^{\mathrm{A}}$
}

\section{Introduction}

The current definition of the term 'evidence-based medicine' coined by Prof Gordon Guyatt, is 'the integration of the best available evidence with clinical expertise and the individual patient's values, preferences, and unique circumstances.'. In India, the paternalistic approach of practising medicine hinders implementing evidencebased dictums in daily clinical practice. ${ }^{2}$ Several misconceptions about evidence-based medicine (EBM) resist its application; for instance, it follows a cook-book approach and fails to account for the patient's perspective. ${ }^{2}$

From the beginning of the COVID-19 pandemic, there have been grave concerns concerning the application of EBM at the global level. The pandemic has questioned the core concepts of EBM; it has demanded a transition to a new era of EBM, 'characterised by scepticism, thoughtfulness, responsiveness and clinically agility in practice.'.

This survey aims to assess the situation of EBM in Mumbai - the city with the highest number of COVID-19 cases in India - and also to evaluate the general perspective of Indian clinicians working in resource-limited conditions towards EBM.

\section{Materials and methods}

This was a multicentric paper-based questionnaire survey carried out in June 2020 among registered medical practitioners working in three dedicated COVID-19 hospitals under the Municipal Corporation of Mumbai (Maharashtra, India) purview. Participation in this survey was voluntary and anonymous. The questions focused on the clinical experience, utilisation of EBM resources, and barriers faced while assimilating the evidence by physicians involved in the treatment of COVID-19 patients. The perspective of physicians towards EBM was also assessed.

\section{Results and discussion}

Out of 400 questionnaires, 213 (53.25\%) were returned. Many respondents $(71.4 \%)$ cited online teaching sessions or discussions

Author: ${ }^{A}$ Urban Primary Health and Training Centre, Mumbai, India
Table 1 . Difficulties faced while assimilating

evidence-based information related to COVID-19

Difficulty faced while assimilating Number, $\mathrm{n}$ Percentage evidence-based information related to COVID-19

Overload of information 114 $53.5 \%$

Unfamiliarity with research methodology

Unfamiliarity with biostatistics

79

$37.1 \%$

Limited access to journal articles

Difficulty in searching relevant 87

medical information

$38 \%$ with colleagues and senior physicians as the primary source of their information related to COVID-19. 41.8\% of respondents used social media platforms such as Facebook, Twitter and WhatsApp to access information, while $26.8 \%$ used medical journals for the same.

There is a considerable prevalence of misconceptions regarding EBM's principles and limitations among the respondents. $42.7 \%$ believe that EBM does not take into account the clinical experience and judgement of a physician. $64.8 \%$ of respondents do not agree that EBM considers patients' values and preferences. $40.8 \%$ feel that EBM advocates a rigid 'cook-book' approach.

Despite the prevailing misconceptions, the majority of the respondents acknowledge the benefits of EBM. $91.5 \%$ of respondents agree that the principles of EBM help them to make decisions about patient care. In addition, $93.4 \%$ clinicians realise the need to broaden their knowledge and skills, to be able to incorporate EBM meticulously in their clinical practice.

\section{Conclusion}

Our survey results highlight the balance maintained between evidence-based medicine and experience-based medicine by Indian physicians. They identify the importance of EBM while acknowledging its shortcomings. They realise the significance of developing their repertoire to understand, appraise, and practise EBM. 
The tremendous surge of information about the SARS-CoV-2 virus has made it difficult for healthcare workers to keep up. It is now more crucial than ever to promote and reinforce the principles of EBM so that we are better prepared for a future pandemic.

\section{Conflicts of interest}

None declared.

\section{References}

1 Siwek J. Evidence-based medicine: common misconceptions, barriers, and practical solutions. Am Fam Physician 2018;98:343-4.

2 Karthikeyan G, Pais P. Clinical judgement and evidence-based medicine: time for reconciliation. Indian J Med Res 2010;132:623-6.

3 Carley S, Horner D, Body R, Mackway-Jones K. Evidence-based medicine and COVID-19: what to believe and when to change. Emerg Med J 2020;37:572-5. 